\title{
RSPH9 methylation pattern as a prognostic indicator in patients with non-muscle invasive bladder cancer
}

\author{
HYUNG-YOON YOON ${ }^{1 *}$, YONG-JUNE KIM ${ }^{2 *}$, JI SANG KIM ${ }^{2}$, YOUNG-WON KIM ${ }^{2}$, HO WON KANG ${ }^{2}$, \\ WON TAE KIM ${ }^{2}$, SEOK-JOONG YUN ${ }^{2}$, KEUN HO RYU ${ }^{3}$, SANG-CHEOL LEE ${ }^{2}$ and WUN-JAE KIM ${ }^{2}$ \\ ${ }^{1}$ Advanced Technology Korea, Cheongju 28637; ${ }^{2}$ Department of Urology, Chungbuk National University College of Medicine, \\ Cheongju 28644; ${ }^{3}$ Database/Bioinformatics Laboratory, Chungbuk National University, Cheongju 28644, Republic of Korea
}

Received August 18, 2015; Accepted October 10, 2015

DOI: $10.3892 /$ or.2015.4409

\begin{abstract}
DNA methylation is a frequent and early epigenetic event with potential application as a biomarker for cancer detection and an indicator of disease evolution. The aim of the present study was to identify novel methylation markers for the prediction of patient outcomes using microarray analysis of DNA methylation in samples from long-term follow-up patients with non-muscle invasive bladder cancer (NMIBC). Candidate methylation markers were selected from our previously published genome-wide methylation profiles. The clinical relevance of candidate methylation markers was determined by quantitative pyrosequencing analysis of 136 human bladder specimens (8 normal controls and 128 NMIBCs). The reversibility of DNA methylation was examined by 5-Aza-CdR treatment in human bladder cancer cell lines. The methylation patterns of candidate markers were significantly associated with aggressive clinicopathological features. In multivariate regression analysis, hypermethylation of radial spoke head 9 homolog (RSPH9) was an independent predictor of disease recurrence (hazard ratio, 3.02; $\mathrm{P}=0.001$ ) and progression (hazard ratio, 8.25; $\mathrm{P}=0.028)$. The methylation level of $R S P H 9$ decreased with
\end{abstract}

Correspondence to: Professor Wun-Jae Kim, Department of Urology, Chungbuk National University College of Medicine, 1 Chungdae-ro, Seowon-gu, Cheongju, Chungbuk 28644, Republic of Korea

E-mail:wjkim@chungbuk.ac.kr

*Contributed equally

Abbreviations: BARHL2, BarH-like homeobox 2; HR, hazard ratio; NC, normal controls; NMIBC, non-muscle invasive bladder cancer; PSQ, pyrosequencing; RAB37, member RAS oncogene family; RSPH9, radial spoke head 9 homolog; TUR, transurethral resection

Key words: methylation, microarray analysis, prognosis, radial spoke head 9 homolog, urinary bladder neoplasms
5-Aza-CdR treatment and progressively increased in its absence in bladder cancer cell lines. RSPH9 methylation is an independent prognostic indicator in NMIBC patients, and could be of value for the assessment of disease recurrence and progression and for clinical decision-making regarding treatment.

\section{Introduction}

Although the majority of newly diagnosed bladder cancer is non-muscle invasive bladder cancer (NMIBC), most patients with NMIBC relapse after adequate treatment, and some progress to muscle invasive disease $(1,2)$. Furthermore, the potential of these tumors for recurrence and progression into muscle invasive disease is highly unpredictable. The detection of high-risk cases after transurethral resection (TUR) of the bladder tumor is essential and depends on efficient prognostic biomarkers for high-risk patients. Research efforts worldwide have focused on the identification of clinically helpful tumor markers or potentially valuable therapeutic targets to improve current diagnostic and management strategies for patients with bladder cancer (3).

Disease-specific aberrant DNA methylation is recognized as a hallmark of many cancers, which led to new opportunities for the understanding, detection, treatment, and prevention of diseases including bladder cancer (4-12). The use of DNA methylation as a biomarker has gained increasing interest in recent years, as aberrant DNA methylation is a major characteristic of bladder cancer and plays a crucial role in tumor initiation and progression (7-13). Although many of the different genetic or epigenetic changes that lead to aberrant gene expression in bladder cancer have been identified, the discovery of novel candidate methylation biomarkers was accelerated by the advent of high-throughput methylation profiling using normal and malignant cells (14).

NMIBC of similar morphology may behave differently, and it is difficult to predict clinical outcomes after initial treatment $(1,2)$. Therefore, novel biomarkers predictive of disease outcomes, in addition to commonly used clinicopathological parameters, would be valuable for guiding appropriate management strategies (7). The aim of the present study was to identify novel methylation markers predictive of patient 
outcomes using microarray analysis of DNA methylation profiles in long-term follow-up NMIBC samples.

\section{Materials and methods}

Subjects and sample collection. A total of 136 human bladder specimens were used for pyrosequencing (PSQ) analyses, including 8 normal controls (NC) and 128 NMIBC samples (Table I). NMIBC specimens were obtained from 128 primary NMIBC patients who underwent TUR for histologically diagnosed transitional cell carcinomas between 1995 and 2010 at our institute. To exclude the possibility of incomplete resection or confounding factors that may unduly affect the analyses, patients followed-up for less than 6 months or those that experienced disease relapse within 6 months were excluded from the study. Samples of normal bladder urothelium obtained from individuals with benign prostate hyperplasia or bladder injury were used as controls.

All tumors were macro-dissected within 15 min of surgical resection. Each NMIBC specimen was confirmed by pathological analysis of a part of the tissue sample (i.e., sections were taken from TUR specimens and then snap-frozen in liquid nitrogen and stored at $-80^{\circ} \mathrm{C}$ ). The specimens were provided by the Chungbuk National University Hospital, a member of the National Biobank of Korea, which is supported by the Ministry of Health, Welfare and Family Affairs. The collection and analysis of all samples was approved by the Chungbuk National University Hospital Institutional Review Board (GR2010-12-010), and informed consent was obtained from each subject.

Tumors were staged according to the 2002 TNM classification and the 1973 WHO grading system (1,2). A second TUR was performed 2-4 weeks after the initial resection if a bladder cancer specimen did not include proper muscle, or if a high-grade tumor was detected. Patients with intermediate- or high-risk NMIBC received one cycle of intravesical therapy. Each patient was followed-up and managed according to the standard recommendations $(1,2)$. Recurrence was defined as the recurrence of primary NMIBC at a lower or equivalent pathologic stage (Ta/T1), and progression was defined as muscular invasion (TNM stage T2 or higher) or metastatic disease.

DNA methylation profiling. Microarray methylation data of 24 human bladder specimens $(\mathrm{NMIBC}=18, \mathrm{NC}=6)$ previously published by our group were used (15). Methylation patterns were assayed using the genome-wide Illumina Infinium HumanMethylation27 BeadChip array (Illumina Inc., San Diego, CA, USA), which enables interrogation of 27,578 CpG dinucleotides covering 14,495 genes. Methylation assays were carried out according to the manufacturer's protocol. Bisulfite conversion of genomic DNA was carried out using the EZ DNA Methylation kit (Zymo Research, Orange, CA, USA). Fluorescence signals corresponding to $\mathrm{C}$ - or T-nucleotides were measured, and the data were used to assign a quantitative measure of methylation level ( $\beta$-value). Each methylation data point represents the fluorescent signal from the methylated (M) and unmethylated (U) alleles. Background intensity was computed from a set of negative controls and subtracted from each analytic data point. The ratio of the fluorescent signals
Table I. Baseline characteristics of the study subjects.

\begin{tabular}{|c|c|c|}
\hline Variables & $\mathrm{NC}(\mathrm{n}=8)$ & $\begin{array}{l}\text { NMIBC } \\
(n=128)\end{array}$ \\
\hline Age (years), mean & $59.0 \pm 22.2$ & $62.6 \pm 14.5$ \\
\hline \multicolumn{3}{|l|}{ Gender, no. of patients $(\%)$} \\
\hline Male & $6(75.0)$ & $103(80.5)$ \\
\hline Female & $2(25.0)$ & $25(19.5)$ \\
\hline \multicolumn{3}{|l|}{ No. of tumors (\%) } \\
\hline Single & - & $77(60.2)$ \\
\hline Multiple & - & $51(39.8)$ \\
\hline \multicolumn{3}{|l|}{ Tumor size, no. of patients (\%) } \\
\hline$<3 \mathrm{~cm}$ & - & $74(57.8)$ \\
\hline$\geq 3 \mathrm{~cm}$ & - & $54(42.2)$ \\
\hline \multicolumn{3}{|l|}{ Grade, no. of patients (\%) } \\
\hline G1 & - & $40(31.3)$ \\
\hline $\mathrm{G} 2$ & - & $75(58.6)$ \\
\hline G3 & - & $13(10.2)$ \\
\hline \multicolumn{3}{|l|}{ T stage, no. of patients (\%) } \\
\hline $\mathrm{Ta}$ & - & $50(39.1)$ \\
\hline $\mathrm{T} 1$ & - & $78(60.9)$ \\
\hline Median RFS, months (range) & - & $30.6(6.0-205.3)$ \\
\hline \multicolumn{3}{|l|}{ Recurrence, no. of patients (\%) } \\
\hline No & - & $74(57.8)$ \\
\hline Yes & - & $54(42.2)$ \\
\hline Median PFS, months (range) & - & $54.1(6.4-205.3)$ \\
\hline Progression, no. of patients (\%) & - & \\
\hline No & - & $113(88.3)$ \\
\hline Yes & - & $15(11.7)$ \\
\hline
\end{tabular}

NC, normal control; NMIBC, non-muscle invasive bladder cancer; RFS, recurrence-free survival; PFS, progression-free survival.

from the 2 alleles was then computed. The $\beta$-value value represents a quantitative measure of the DNA methylation level of specific $\mathrm{CpG}$ islands, and ranges from 0 (completely unmethylated) to 1 (completely methylated).

PSQ analysis. The DNA methylation status of candidate NMIBC-specific hypermethylated $\mathrm{CpG}$ sites was assessed by PSQ using PyroMark Q96 ID (Qiagen, Valencia, CA, USA) according to the manufacturer's instructions. PSQ primers were designed to encompass the $\mathrm{CpG}$ sites assayed on the Illumina Infinium array. The primer sequences and amplification conditions are described in Table II.

Bladder cancer cell lines, culture, and drug treatment. The human bladder cancer cell lines T24 (KCLB 30004) and J82 (KCLB 30001) were purchased from the Korean Cell Line Bank (Seoul, Korea). All cells were maintained in RPMI-1640 medium supplemented with 10\% FBS (both from Gibco-BRL, Grand Island, NY, USA) and L-glutamine, and cells were incubated in a humidified atmosphere with $5 \% \mathrm{CO}_{2}$ at $37^{\circ} \mathrm{C}$. Both cell lines were cultured until confluent and then treated 
with PBS or $0.3 \mu \mathrm{mol} / 1$ 5-Aza-CdR (both from Sigma-Aldrich, St. Louis, MO, USA). The medium was changed the following day, and cells were counted and harvested on days 3 and 7, and then once per week.

Statistical analysis. DNA methylation data were normalized using quantile normalization in the $\mathrm{R}$ language environment (version 2.10.0, available at http://www.r-project.org/). The detailed analytical methods have been described previously $(16,17)$. To detect NMIBC-specific candidate methylation markers, genes whose methylation levels differed between NMIBC and NC by a $\beta$-value $(\Delta \beta$-values) $>0.5$ were selected. To validate the genes identified in this study, our $\Delta \beta$-values were compared with those of two microarray data sets obtained from Western populations (9,13): i), 32 bladder tissues (NMIBC $=26, \mathrm{NC}=6$ ); and ii), 70 bladder tissues (NMIBC $=64, \mathrm{NC}=6)$. Continuous variables between groups were compared using a two-sample t-test or ANOVA trend analysis using polynomial contrasts. To minimize bias against arbitrary cut-off points, median values were applied to divide patients into subgroups (hypomethylation or hypermethylation), and the survival function of candidate genes was evaluated. The Kaplan-Meier method was used to estimate the time-to-recurrence or progression based on methylation status, and differences were assessed using log-rank statistics. For the multivariate Cox proportional hazards regression models, the prognostic value of methylation status was evaluated separately and adjusted for well-known clinicopathological (gender, age, tumor size, tumor number, intravesical therapy, grade, and stage) factors. Statistical analysis was performed using IBM SPSS Statistics ver. 21.0 (IBM Co., Armonk, NY, USA). $\mathrm{P}<0.05$ was considered statistically significant.

\section{Results}

Baseline characteristics. The baseline characteristics of the NC and NMIBC patients are presented in Table I. Mean recurrence-free survival and progression-free survival in patients with NMIBC was 44.1 339.1 months (median, 30.6; range, 6.0-205.3) and 60.8 40.7 months (median, 54.1; range, 6.4-205.3), respectively.

Identification of differentially methylated and expressed genes in NMIBC and NC. Our previously published genome-wide methylation profiles obtained from 18 NMIBC patients were analyzed and compared with those from $6 \mathrm{NC}$ (15). The complete sets of microarray data derived from the human bladder tissues are available online (http://www.ncbi.nlm.nih. gov/geo/) under the data series accession number GSE37817. To select NMIBC-specific methylation markers, a highly stringent selection criterion $(\Delta \beta$-value $>0.5)$ was applied. We identified 25 unique $\mathrm{CpG}$ island loci in 23 genes that were hypermethylated in NMIBC compared with NC, and these are listed in Table III. The validity of our candidate genes as methylation markers for NMIBC was evaluated using an independent set of Infinium microarray methylation data derived from two Western populations $(9,13)$. Only partial validation was possible because of the limited data provided in both studies. The $\Delta \beta$-values obtained in the present study were comparable to those of other studies (Table III). Of the 


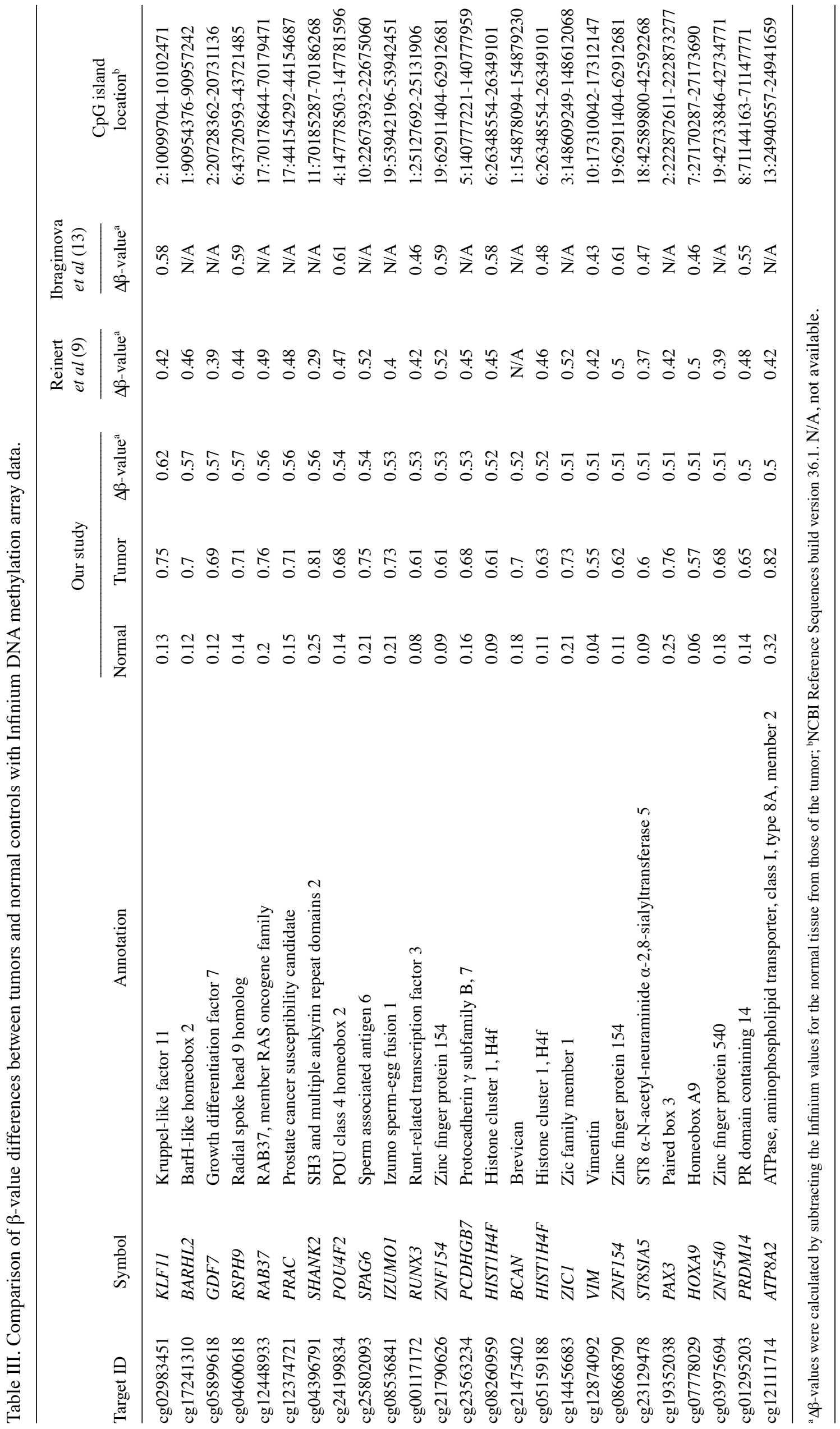


Table IV. Association between methylation markers and clinicopathological characteristics.

\begin{tabular}{|c|c|c|c|c|c|c|}
\hline \multirow[b]{2}{*}{ Variables } & \multicolumn{2}{|l|}{ BARHL2 } & \multicolumn{2}{|l|}{ RSPH9 } & \multicolumn{2}{|l|}{$R A B 37$} \\
\hline & Methylation level (\%) & P-value & Methylation level (\%) & P-value & Methylation level (\%) & P-value \\
\hline Normal vs. cancer & & $<0.001^{\mathrm{a}}$ & & $<0.001^{\mathrm{a}}$ & & $<0.001^{\mathrm{a}}$ \\
\hline Normal & $17.1 \pm 3.5$ & & $18.0 \pm 6.6$ & & $24.4 \pm 1.0$ & \\
\hline Cancer & $53.8 \pm 24.0$ & & $52.6 \pm 25.9$ & & $42.6 \pm 18.2$ & \\
\hline No. of tumors & & $0.043^{\mathrm{a}}$ & & $0.030^{\mathrm{a}}$ & & $0.496^{\mathrm{a}}$ \\
\hline Single & $50.5 \pm 25.3$ & & $48.6 \pm 25.6$ & & $43.5 \pm 18.4$ & \\
\hline Multiple & $58.9 \pm 21.2$ & & $58.7 \pm 25.4$ & & $41.2 \pm 18.0$ & \\
\hline Tumor size $(\mathrm{cm})$ & & $0.461^{\mathrm{a}}$ & & $0.196^{\mathrm{a}}$ & & $0.386^{\mathrm{a}}$ \\
\hline$<3$ & $52.4 \pm 25.2$ & & $55.1 \pm 25.0$ & & $41.4 \pm 17.8$ & \\
\hline$\geq 3$ & $55.7 \pm 22.4$ & & $49.1 \pm 26.0$ & & $44.2 \pm 18.7$ & \\
\hline Grade & & $0.002^{\mathrm{b}}$ & & $0.001^{\mathrm{b}}$ & & $0.753^{\mathrm{b}}$ \\
\hline G1 & $46.7 \pm 23.4$ & & $45.6 \pm 22.8$ & & $45.7 \pm 23.4$ & \\
\hline $\mathrm{G} 2$ & $54.7 \pm 23.0$ & & $52.1 \pm 27.0$ & & $39.6 \pm 17.6$ & \\
\hline G3 & $70.8 \pm 23.5$ & & $76.6 \pm 11.5$ & & $49.6 \pm 20.2$ & \\
\hline T stage & & $0.004^{\mathrm{a}}$ & & $0.042^{\mathrm{a}}$ & & $0.216^{\mathrm{a}}$ \\
\hline $\mathrm{Ta}$ & $46.2 \pm 24.1$ & & $47.7 \pm 23.1$ & & $45.1 \pm 20.0$ & \\
\hline $\mathrm{T} 1$ & $58.7 \pm 22.7$ & & $57.8 \pm 27.2$ & & $41.0 \pm 17.1$ & \\
\hline Recurrence & & $0.048^{\mathrm{a}}$ & & $0.005^{\mathrm{a}}$ & & $0.154^{\mathrm{a}}$ \\
\hline No & $50.5 \pm 24.4$ & & $47.1 \pm 24.3$ & & $40.5 \pm 16.1$ & \\
\hline Yes & $58.7 \pm 22.7$ & & $60.1 \pm 26.4$ & & $45.4 \pm 20.5$ & \\
\hline Progression & & $0.022^{\mathrm{a}}$ & & $0.002^{\mathrm{a}}$ & & $0.554^{\mathrm{a}}$ \\
\hline No & $52.1 \pm 24.2$ & & $50.1 \pm 25.1$ & & $43.0 \pm 25.1$ & \\
\hline Yes & $67.1 \pm 18.6$ & & $71.5 \pm 24.4$ & & $40.0 \pm 23.5$ & \\
\hline
\end{tabular}

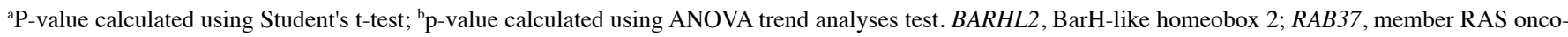
gene family; $R S P H 9$, radial spoke head 9 homolog.

candidate markers identified, genes in the top fifth $\Delta \beta$-value were selected for evaluation of their clinical relevance in the validation cohort.

$P S Q$ analysis. To verify the clinical relevance of candidate methylation markers, PSQ analysis was performed using bisulfite-modified genomic DNA obtained from 136 human bladder specimens (NMIBC=128, NC=8). PSQ analysis was technically possible in three out of five candidate genes [BarH-like homeobox 2 (BARHL2), radial spoke head 9 homolog (RSPH9), and member RAS oncogene family (RAB37)], and these genes were analyzed by PSQ in the present study.

Association between methylation levels and clinicopathological variables. As shown in Table IV, the methylation levels of candidate genes were significantly higher in NMIBC patient samples than in normal samples $(\mathrm{P}<0.001)$. To evaluate the relationship between methylation patterns and clinicopathological factors, methylation levels were examined in correlation with well-known prognostic factors such as tumor number, tumor size, and tumor grade and stage. The results showed that high levels of methylation of BARHL2 and RSPH9 were significantly associated with tumor size, grade, and stage.
Methylation status as a predictor of prognosis. The methylation levels of $B A R H L 2$ and $R S P H 9$ were significantly higher in the poor prognosis group (recurrence or progression) than in the favorable prognosis group (Table IV). Stratification of NMIBC patients into three prognostic groups (non-recurrence and nonprogression, recurrence only, and progression) showed that the methylation of BARHL2 and RSPH9 was positively correlated with poor prognosis (Fig. 1). To further determine the relevance of candidate gene methylation status as a predictive indicator, the methylation levels of each gene were dichotomized (hypomethylation or hypermethylation) using median cut-off points. Kaplan-Meier estimates identified significant differences in time-to-recurrence or progression according to methylation status of BARHL2 and RSPH9 (Fig. 2, log-rank test, each $\mathrm{P}<0.05)$. In univariate and multivariate Cox regression analyses, RSPH9 methylation status was an independent predictor of recurrence [hazard ratio $(\mathrm{HR}), 3.02 ; \mathrm{P}=0.001$ ] and progression (HR, $8.25 ; \mathrm{P}=0.028$, Table $\mathrm{V}$ ) in primary NMIBC patients. However, differences in BARHL2 methylation did not reach statistical significance for the prediction of prognosis $(\mathrm{P}>0.05)$.

Reversibility of RSPH9 methylation with AZA treatment. To investigate the potential reversibility of RSPH9 methylation, 

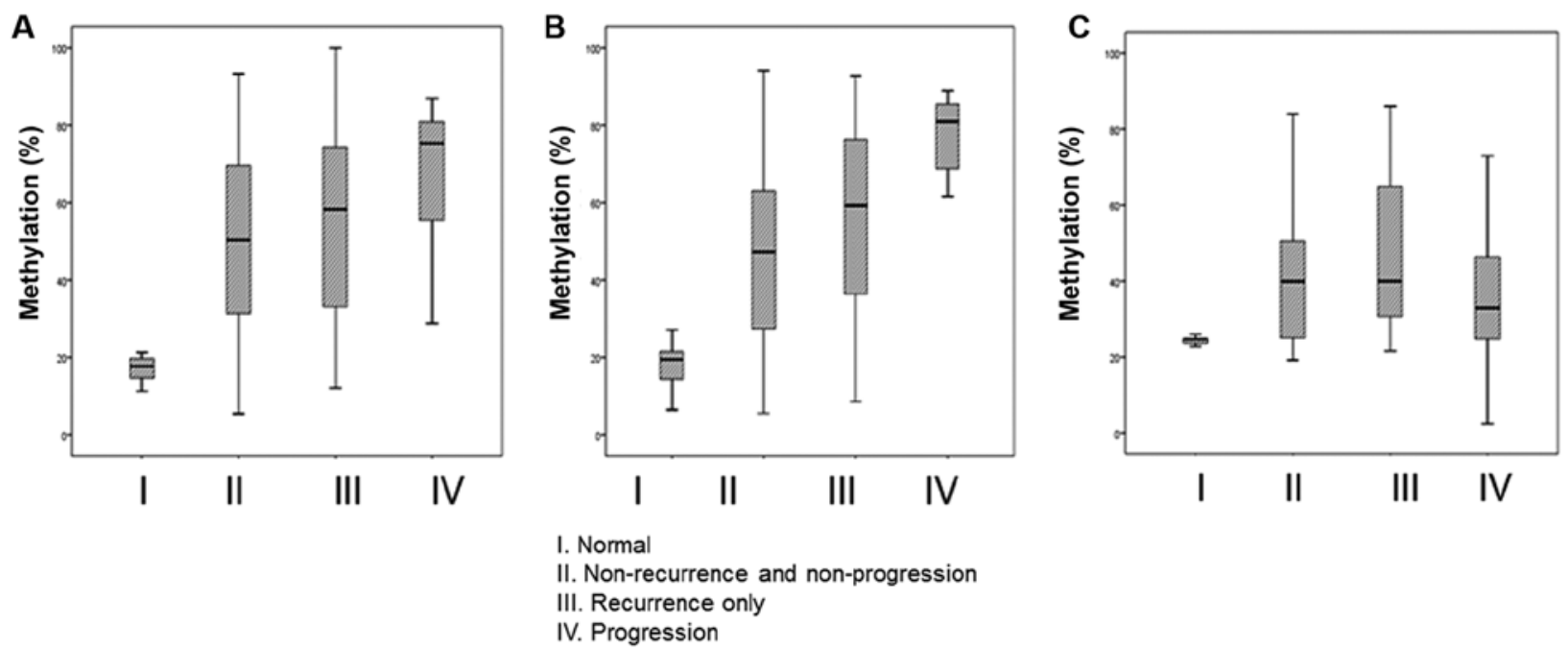

Figure 1. Methylation level of BarH-like homeobox 2 (BARHL2) (A), radial spoke head 9 homolog (RSPH9) (B), and member RAS oncogene family $(R A B 37)(C)$ in bladder tissues according to the prognosis of the patients.
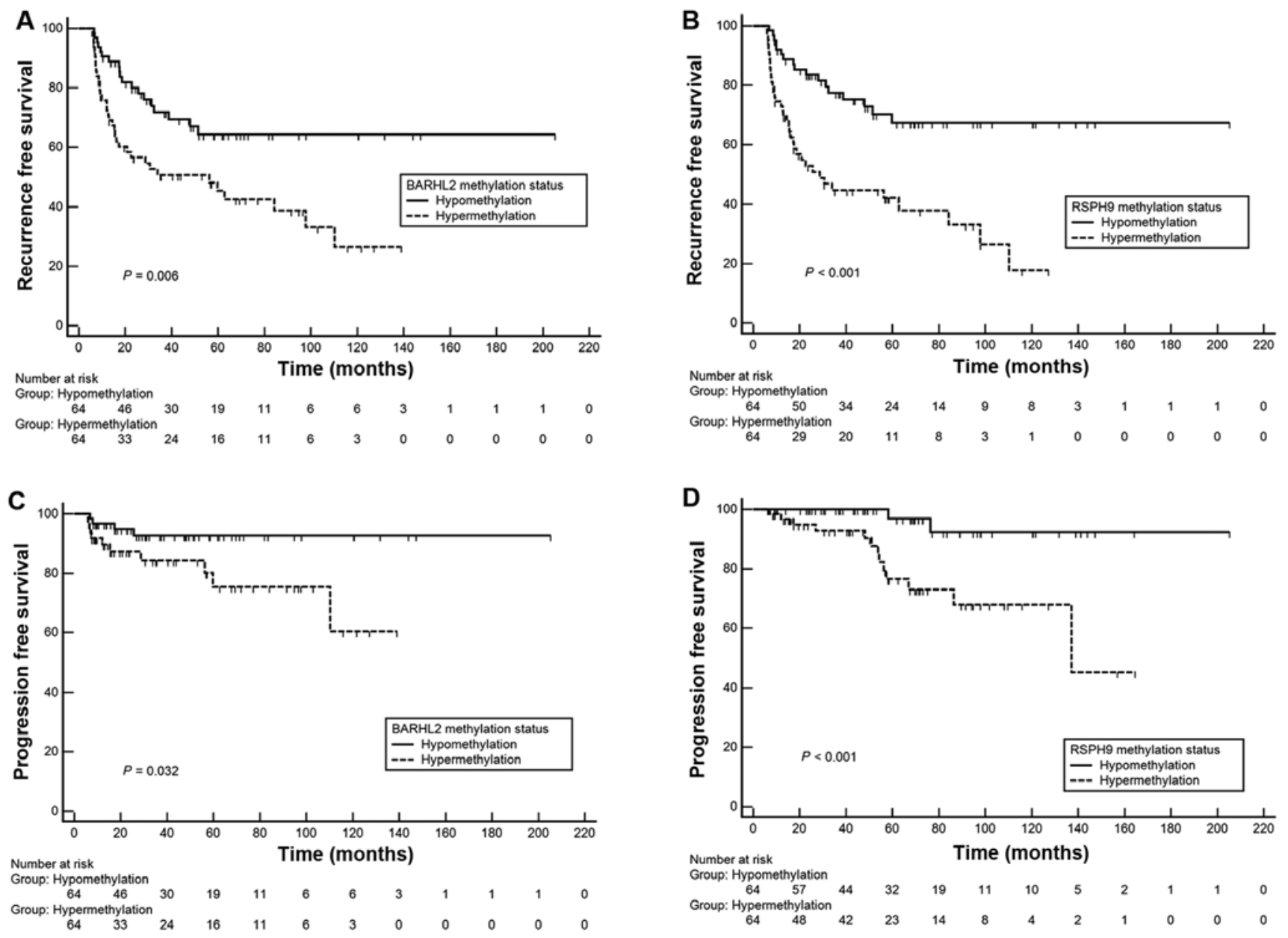

Figure 2. Kaplan-Meier curves for the prediction of prognosis according to the methylation status of each gene in patients with non-muscle invasive bladder cancer (NMIBC). Recurrence-free survival (A and B) and progression-free survival (C and D) according to the methylation status of BARHL2 and RSPH9.

cells from two bladder cancer lines (T24 and J82) were treated with $0.3 \mu \mathrm{M}$ 5-Aza-CdR for $24 \mathrm{~h}$, and sequential changes in cell number and $R S P H 9$ methylation level were evaluated in each cell line. Compared to the untreated cells, a decrease in cell count was detected on day 1 after 5-Aza-CdR treatment, reaching a maximal level on day 3 in the J82 and T24 cells, followed by a gradual increase in cell number starting 1 week after 5-Aza-CdR treatment, reaching constant values after 6 weeks in both cell lines. Consistent with the cell-count changes, the methylation level of RSPH9 decreased on day 3 
Table V. Multivariate Cox regression analysis of disease outcomes according to RHPH9 methylation in non-muscle invasive bladder cancer $(n=128)$.

\begin{tabular}{|c|c|c|c|c|}
\hline \multirow[b]{2}{*}{ Variables } & \multicolumn{2}{|c|}{ Recurrence } & \multicolumn{2}{|c|}{ Progression } \\
\hline & HR $(95 \% \mathrm{CI})$ & P-value & $\operatorname{HR}(95 \% \mathrm{CI})$ & P-value \\
\hline Age ( $<66$ vs. $\geq 66$ years) & $0.99(0.57-1.76)$ & 0.973 & $2.31(0.69-7.73)$ & 0.175 \\
\hline Gender (male vs. female) & $0.56(0.25-1.26)$ & 0.158 & $0.18(0.20-1.55)$ & 0.118 \\
\hline No. of tumors (single vs. multiple) & $1.14(0.62-2.08)$ & 0.672 & $6.91(1.70-28.10)$ & 0.007 \\
\hline Tumor size $(<3 \mathrm{vs} . \geq 3 \mathrm{~cm})$ & $1.27(0.69-2.23)$ & 0.158 & $2.92(0.85-9.96)$ & 0.088 \\
\hline Stage (Ta vs. T1) & $0.69(0.33-1.43)$ & 0.322 & $1.17(0.12-11.87)$ & 0.896 \\
\hline Grade & & & & 0.021 \\
\hline G1 & 1 & - & 1 & - \\
\hline $\mathrm{G} 2$ & $1.37(0.65-2.90)$ & 0.408 & $3.04(0.23-40.06)$ & 0.399 \\
\hline G3 & $1.20(0.41-3.53)$ & 0.744 & $17.49(1.11-276.29)$ & 0.042 \\
\hline Intravesical therapy (no vs. yes) & $1.41(0.70-2.83)$ & 0.331 & $0.84(0.14-4.91)$ & 0.835 \\
\hline RSPH9 (hypomethylation vs. hypermethylation) & $3.02(1.61-5.67)$ & 0.001 & $8.25(1.26-54.09)$ & 0.028 \\
\hline
\end{tabular}

RSPH9, radial spoke head 9 homolog.

A

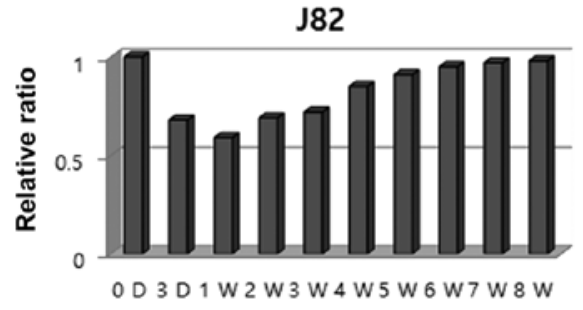

C

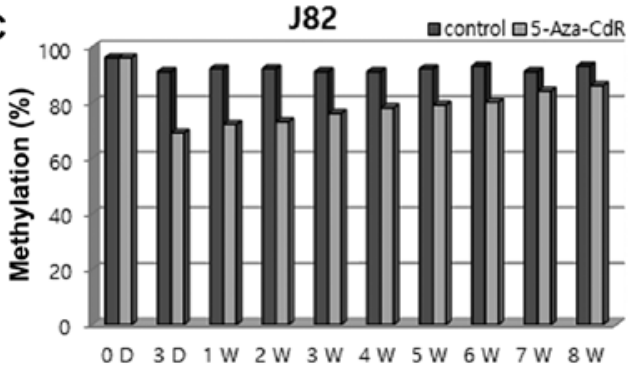

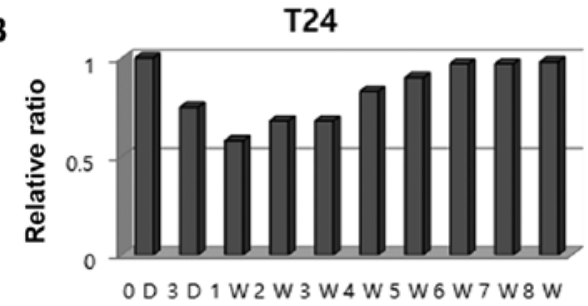

D

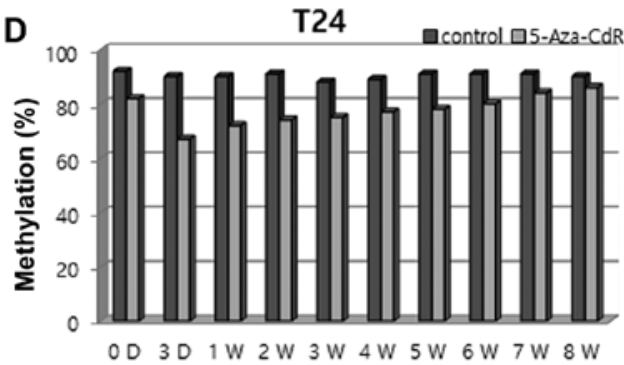

Figure 3. Changes of tumor cell count and RSPH9 methylation level in bladder cancer cell lines treated with 5-Aza-CdR. Tumor cell count decreased initially in response to 5-Aza-CdR treatment and increased gradually over time without Aza-CdR in J82 (A) and T24 (B) cells. The methylation level of RSHP9 changed in correlation with cell-count changes in the J82 (C) and T24 (D) cell lines. Cell count was represented as the relative ratio of the number of treated to untreated cells.

and progressively increased 1 week after treatment in the J82 and T24 cell lines (Fig. 3).

\section{Discussion}

Similar to other human cancers, bladder cancer is a molecular disease driven by multiple genetic, epigenetic, and environmental factors $(6,7)$. DNA methylation, which is the most common and best characterized epigenetic change in bladder cancer, inactivates tumor-suppressor genes and may be used as potential biomarkers $(6,7)$. In the present study, we used microarray-based profiling to discover novel epigenetic markers relevant to NMIBC. Among the candidate methylation markers identified, the RSPH9 methylation pattern showed close associations with aggressive NMIBC characteristics, including advanced stage and high tumor grade. The methylation status of $\mathrm{RSPH} 9$ was identified as an independent predictive indicator of prognosis.

The evolution of classic single-gene DNA methylation detection assays to genome-wide microarray-based analyses enabled a better understanding of the role of DNA methylation in cancer $(6,14)$. However, the identification of bona fide candidate methylation markers of clinical relevance requires appropriate selection criteria, validation with external data sets, 
confirmation of the methylation status using highly targeted locus-specific assays of human tissues, and comparison with clinicopathological parameters or disease outcomes $(4,18)$. Considering these criteria, the results of the present study are promising. Candidate methylation markers were selected by genome-wide microarray profiling, and their relevance was validated with two microarray data sets obtained from Western populations with NMIBC $(9,13)$. Furthermore, the association between the methylation status of RSPH 9 and prognostic outcomes was verified in long-term follow-up NMIBC patients. The results suggested that the novel methylation marker identified is specific to NMIBC and appropriate for predicting prognosis.

Little information is available concerning the function of the RSPH9 gene (19-21). It is located on chromosome 6p21.1 and encodes radial spoke head protein 9. Previous studies suggested that $R S P H 9$ is mutated in primary ciliary dyskinesia patients with microtubule defects (19-21). To the best of our knowledge, the present study is the first to identify $R S P H 9$ as a cancer-related methylation marker. Despite the prognostic significance of RSPH9 in NMIBC identified in the present study, these findings do not indicate that it plays a crucial role in bladder tumor initiation or progression. The lack of a clear association between these candidate markers and bladder cancer is a limitation of the present study, and this issue will be addressed in future studies. However, the objective of the present study was the identification of disease markers; we focused on the association between methylation changes of specific methylation markers and disease phenotype rather than analyzing the effect of methylation status on gene transcription and function (4).

DNA methylation is a reversible modification, which makes it a potential therapeutic target. Drugs that target epigenetic alterations, such as DNA methylation inhibitors, restore the activity of genes by targeting aberrant heterochromatic regions, ultimately leading to the reactivation of tumor-suppressor genes and/or other genes that are crucial for normal cellular function (5). In the present study, we showed that the methylation level of RSPH 9 in human bladder cancer cell lines decreased in response to 5-Aza-CdR treatment and increased progressively in its absence. The reversibility of RSPH 9 methylation shown in the present study confirms its value as a candidate therapeutic target. Large-scale validation studies using human samples, as well as functional analyses and a gene ontologic approach to the study of $R S P H 9$, may provide additional knowledge about its biological mechanism and clinical relevance.

From a clinical point of view, the most promising applications for epigenetic markers are early detection, prediction of response to treatment, and indication of disease prognosis. The results presented herein are promising because the candidate methylation markers were selected from a genome-wide analysis and validated in a relatively large number of human tissue samples obtained from long-term follow-up patients. In addition, the selected methylation markers are independent predictors of disease outcome. An accurate prediction of prognosis made using these candidate methylation markers would aid clinicians in terms of patient counseling, determining the frequency and extent of monitoring, and whether more aggressive therapy is needed. However, despite these promising results, further validation studies are necessary to reduce false prediction rates and achieve reliable clinical relevance. It may also lead to new therapies that target specific molecular defects, thereby significantly lowering the morbidity associated with NMIBC.

In conclusion, our findings suggest that the novel methylation marker RSPH9 is an independent indicator of prognosis in NMIBC patients. This prognostic marker may constitute a promising tool for assessing the recurrence and progression of NMIBC and may facilitate the design of individualized therapeutic modalities.

\section{Acknowledgements}

The present study was supported by the Basic Science Research Program through the National Research Foundation of Korea (NRF) funded by the Ministry of Education (2011-0023308) and Ministry of Science, ICT and Future Planning (NRF-2014R1A2A1 A09006983). All samples derived from the National Biobank of Korea were obtained with informed consent under institutional review boardapproved protocols.

\section{References}

1. Babjuk M, Burger M, Zigeuner R, Shariat SF, van Rhijn BW, Compérat E, Sylvester RJ, Kaasinen E, Böhle A, Palou Redorta J, et al; European Association of Urology: EAU guidelines on non-muscle-invasive urothelial carcinoma of the bladder: Update 2013. Eur Urol 64: 639-653, 2013.

2. Kamat AM, Hegarty PK, Gee JR, Clark PE, Svatek RS, Hegarty N, Shariat SF, Xylinas E, Schmitz-Dräger BJ, Lotan Y, et al; International Consultation on Urologic Disease - European Association of Urology Consultation on Bladder Cancer 2012: ICUD-EAU International Consultation on Bladder Cancer 2012: Screening, diagnosis, and molecular markers. Eur Urol 63: 4-15, 2013.

3. Cheng L, Davison DD, Adams J, Lopez-Beltran A, Wang L, Montironi R and Zhang S: Biomarkers in bladder cancer: Translational and clinical implications. Crit Rev Oncol Hematol 89: 73-111, 2014.

4. Ushijima T: Detection and interpretation of altered methylation patterns in cancer cells. Nat Rev Cancer 5: 223-231, 2005.

5. Baylin SB and Jones PA: A decade of exploring the cancer epigenome - biological and translational implications. Nat Rev Cancer 11: 726-734, 2011

6. Besaratinia A, Cockburn M and Tommasi S: Alterations of DNA methylome in human bladder cancer. Epigenetics 8: 1013-1022, 2013.

7. Kim WJ and Kim YJ: Epigenetic biomarkers in urothelial bladder cancer. Expert Rev Mol Diagn 9: 259-269, 2009.

8. Kim EJ, Kim YJ, Jeong P, Ha YS, Bae SC and Kim WJ: Methylation of the RUNX3 promoter as a potential prognostic marker for bladder tumor. J Urol 180: 1141-1145, 2008.

9. Reinert T, Modin C, Castano FM, Lamy P, Wojdacz TK, Hansen LL, Wiuf C, Borre M, Dyrskjøt L and Orntoft TF: Comprehensive genome methylation analysis in bladder cancer: Identification and validation of novel methylated genes and application of these as urinary tumor markers. Clin Cancer Res 17: 5582-5592, 2011.

10. Sánchez-Carbayo M: Hypermethylation in bladder cancer: Biological pathways and translational applications. Tumour Biol 33: 347-361, 2012.

11. Kandimalla R, van Tilborg AA and Zwarthoff EC: DNA methylation-based biomarkers in bladder cancer. Nat Rev Urol 10: 327-335, 2013.

12. Vinci S, Giannarini G, Selli C, Kuncova J, Villari D, Valent F and Orlando C: Quantitative methylation analysis of BCL2, hTERT, and DAPK promoters in urine sediment for the detection of non-muscle-invasive urothelial carcinoma of the bladder: A prospective, two-center validation study. Urol Oncol 29: 150-156, 2011. 
13. Ibragimova I, Dulaimi E, Slifker MJ, Chen DY, Uzzo RG and Cairns P: A global profile of gene promoter methylation in treatment-naïve urothelial cancer. Epigenetics 9: 760-773, 2014.

14. Kalari S and Pfeifer GP: Identification of driver and passenger DNA methylation in cancer by epigenomic analysis. Adv Genet 70: 277-308, 2010.

15. Kim YJ, Yoon HY, Kim JS, Kang HW, Min BD, Kim SK, Ha YS, Kim IY, Ryu KH, Lee SC, et al: HOXA9, ISL1 and ALDH1A3 methylation patterns as prognostic markers for nonmuscle invasive bladder cancer: Array-based DNA methylation and expression profiling. Int J Cancer 133: 1135-1142, 2013.

16. Lee JS, Leem SH, Lee SY, Kim SC, Park ES, Kim SB, Kim SK, Kim YJ, Kim WJ and Chu IS: Expression signature of E2F1 and its associated genes predict superficial to invasive progression of bladder tumors. J Clin Oncol 28: 2660-2667, 2010.

17. Kim WJ, Kim EJ, Kim SK, Kim YJ, Ha YS, Jeong P, Kim MJ, Yun SJ, Lee KM, Moon SK, et al: Predictive value of progression-related gene classifier in primary non-muscle invasive bladder cancer. Mol Cancer 9: 3, 2010.

18. Olkhov-Mitsel E and Bapat B: Strategies for discovery and validation of methylated and hydroxymethylated DNA biomarkers. Cancer Med 1: 237-260, 2012.
19. Castleman VH, Romio L, Chodhari R, Hirst RA, de Castro SC, Parker KA, Ybot-Gonzalez P, Emes RD, Wilson SW, Wallis C, et al: Mutations in radial spoke head protein genes RSPH9 and RSPH4A cause primary ciliary dyskinesia with central-microtubular-pair abnormalities. Am J Hum Genet 84: 197-209, 2009.

20. Onoufriadis A, Shoemark A, Schmidts M, Patel M, Jimenez G, Liu H, Thomas B, Dixon M, Hirst RA, Rutman A, et al: Targeted NGS gene panel identifies mutations in RSPH1 causing primary ciliary dyskinesia and a common mechanism for ciliary central pair agenesis due to radial spoke defects. Hum Mol Genet 23: 3362-3374, 2014

21. Ziętkiewicz E, Bukowy-Bieryłło Z, Voelkel K, Klimek B, Dmeńska H, Pogorzelski A, Sulikowska-Rowińska A, Rutkiewicz E and Witt M: Mutations in radial spoke head genes and ultrastructural cilia defects in East-European cohort of primary ciliary dyskinesia patients. PLoS One 7: e33667, 2012. 